\title{
Guest Editorial: Emotion in Games
}

\author{
Georgios N. Yannakakis, Katherine Isbister, Ana Paiva and Kostas Karpouzis
}

\section{EMOTION IN GAMES}

C OMPUTER games can challenge affective interaction at large for a number of reasons: they arguably define the richest and most dynamic form of human computer interaction; they may elicit complex patterns of emotion at multiple and diverse facets of player immersion; they are content-intensive environments offering rich and variant contextual building blocks for player experience; they can realize game-player interaction via a plethora of input modalities; and finally, they offer rich social interaction via their multi-player capacities.

Because of all above challenges, emotion research in and through games has resulted to the advancement of the field of affective interaction. Studies on emotion in games have offered brand new approaches, methods and solutions at all basic steps of the affective loop (elicitation, modeling, expression, adaptation). In particular, emotion has been linked to and elicited via different content types in a game; methods such as sequence mining, preference learning and deep learning have been introduced through games offering solutions to the challenges faced within feature extraction and affect modeling; new rank-based methods have been introduced to eliminate biases in player experience annotation; and, new frameworks - such as the experience-driven procedural content generation framework - have been introduced to represent aspects of affect adaptation.

Finally, games can best realize affective interaction. Their rich and multifaceted context (e.g. agents, music, sound effects, audio, virtual cinematography, game mechanics) offers unique stimuli for eliciting emotion. Their users (i.e. players) are more than happy to provide emotion researchers with multiple modalities of input as long as doing so will augment their player experience.

- G. N.. Yannakakis is with the Institute of Digital Games, University of Malta, Msida 2080, Malta.

E-mail: georgios.yannakakis@um.edu.mt

- K. Isbister is with the Game Innovation lab, NYU Polytechnic School of Engineering, Brooklyn, NY, US.

E-mail: isbister@duke.poly.edu

- A. Paiva is with the Intelligent Agents and Synthetic Characters Group, INESC-ID, Portugal.

E-mail: ana.paiva@inesc-id.pt

- K. Karpouzis is with the Image, Video and Multimedia Systems Lab, National Technical University of Athens, Greece.

E-mail:kkarpou@cs.ntua.gr
As games are designed to offer affective experiences which are influenced by player feedback, players are willing to go through e.g. frustrating, anxious, and fearful episodes of play for experiencing involvement and powerful emotional gaming. To that end, a player more than any other form of human computer interaction - is generally open to affective-based alterations of the interaction and influences of his/her emotional state

The purpose of this special issue on Emotion in Games was to bring together contributions from specialists in affective computing, artificial intelligence, user experience research and multimodal interfaces that will advance the state-of-the-art in player experience research; affect induction, sensing and modeling; and affect-driven game adaptation. That was reflected on the range of papers submitted. A total of twenty two papers were submitted for this special issue which underwent a thorough and highly-selective process of peer review and revision, yielding the four papers accepted for publication.

\section{The Papers in this Special Issue}

This special issue features four papers: the first three revolve around the area of affective game agents whereas the fourth one investigates the impact of affect and engagement on game-based learning. In our opening paper, The Mind Module - Using an Affect and Personality Computational Model as a Game-play Element, Eladhari introduces a modular computational model for capturing affective and personality aspects of game agents. The module is tested on a number of dissimilar game prototypes. Within the topic of affective agents, Bosse and Zwanenburg, in their paper Do Prospect-Based Emotions Enhance Believability of Game Characters? A Case Study in the Context of a Dice Game, enrich the belief-desireintention model with affective capacities. The model is evaluated with respect to its ability to enhance the believability of game agents and player involvement in two simple test-bed games. Popescu et al. in GAMYGDALA: an Emotion Engine for Games present GAMYGDALA, an emotional appraisal engine that enables game designers equipping non-player characters with emotive capabilities. The engine is built on the OCC model and is tested on four games (an arcade game, a role playing game, a real-time strategy game and a firstperson shooter) and a multi-NPC simulation supporting both the generalizability and the scalability of the engine. 
Finally, in Affect and Engagement in Game-Based Learning Environments, Sabourin and Lester provide a thorough analysis of the relationships between affect, engagement and learning within games. They test their hypotheses in the Crystal Island game and their findings suggest that game-based learning environments such as the one tested can assist learning activities through enhanced engagement and positive affect.

\section{Summary and FUtUre Directions}

This issue draws together a number of case studies that demonstrate the application of affective computing within the games domain. The papers appearing in the issue offer a mere instance of the ongoing investigations on the topic of emotion in games and the growing academic interest in the field of player experience. The recent boost of academic interest in the topic is demonstrated by the increasing number of relevant papers published (as well as relevant workshops and special sessions organized) in the key venues of game research (e.g. FDG, IEEE CIG, AIIDE and CHI) and emotion research (e.g. ACII and ICMI). In addition, that interest is coupled with an increasing trend towards the use of sophisticated methods for emotion annotation, feature extraction, affect modeling and adaptation within the challenging domain of games; arguably, games and emotion were not even considered in the early days of conferences on emotion and game research, respectively.

Future directions for research in this area include [1]: (a) The human-machine co-creation approach is getting increasingly important for game design and the potential of emotion-driven, mixed-initiative design needs to be investigated in depth. (b) The impact of emotion in game development can be evident and in all phases of game production and future research needs to focus at establishing protocols for the integration of emotion research in the pipeline of game production. (c) The study of emotion in games as represented by the affective computing community can only benefit from stronger links to and collaborations with adjacent research fields which include the areas of game studies, game design, user and user experience research, and experimental psychology. (d) The use of procedural content generation techniques for the design of better games has reached a peak of interest in commercial and independent game development which is showcased by successful (almost entirely procedurally generated) games such as Minecraft (Mojang, 2011). The automation of content creation offers a unique opportunity towards realizing affect-driven content generation in games. (e) Recent advances in sensor technology have resulted in low-cost unobtrusive biofeedback devices appropriate for gaming applications and recent technology advances in gaming peripherals such as the PrimeSense camera showcase a promising future for multimodal natural interaction in games. (f) Massive sets of player metrical data (metrics) is currently available and analyzed, thus, empowering the design of future games. While such data usually contains behavioral aspects of playing experience, data mining and data analysis research will need to focus on inferring the relationship between detailed player metrics, and cognitive and affective maps of experience.

\section{References}

[1] G. N. Yannakakis and A. Paiva, "Emotion in Games," Handbook of Affective Computing, 2014.

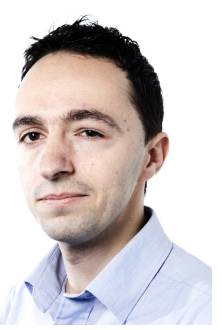

Georgios N. Yannakakis (S04-M05) is an Associate Professor at the Institute of Digital Games, University of Malta. He does research at the crossroads of $\mathrm{Al}$, affective computing, advanced game technology, and computational creativity. He has published over 150 journal and international conference papers in the aforementioned fields. His research has been funded by several national and EU grants and has appeared in Science Magazine and New Scientist among other venues. He is an Associate Editor of the IEEE TRANS. ON AFFECTIVE COMPUTING and the IEEE TRANS. on COMPUtational InTElligence AND AI in GAMES.

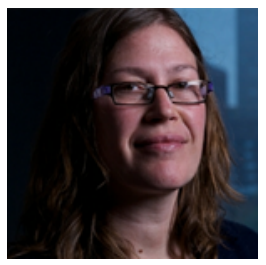

Katherine Isbister is director of the Game Innovation Lab at NYU Polytechnic School of Engineering, serves on the Presidium of the MAGNET center, and is jointly appointed as a faculty member of the NYU Game Center. She has written two books: Better Game Characters by Design and Game Usability. Better Game Characters was nominated for a Game Developer Magazine Frontline Award. Isbister serves on the Editorial Board of the International Journal Member of the ACM. of Human Computer Studies, and is a Senior

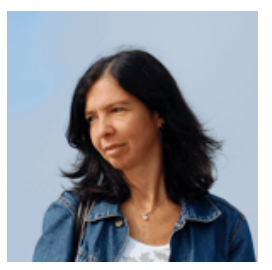

Ana Paiva is Professor in the Department of Computer Science and Engineering of Instituto Superior Tcnico from the Technical University of Lisbon. She is also the group leader of GAIPS, a research group on agents and synthetic characters at INESC-ID. Her main scientific interests lay in the area of Autonomous Agents, Embodied Conversational Agents and Robots and Multiagent Simulation Systems. Prof. Paiva has been researching in the area of artificial intelligence for the past twenty years.

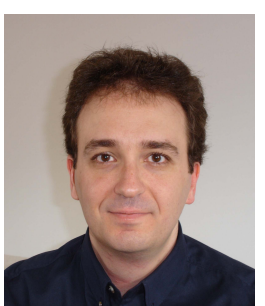

Kostas Karpouzis (M07-SM12) is an associate researcher at the Image, Video and Multimedia System Lab of the Institute of Communication and Computer Systems in Athens, Greece. His research interests include natural interaction, games-based learning and serious games, and emotion recognition. He was an active contributor to the "The HUMAINE Handbook: EmotionOriented Systems" and the "Blueprint for Affective Computing". Since 2010, he is the Student Activities Chair for IEEE Greece. He was the Technical Manager of the FP7 Siren project, voted Best Learning Game in Europe in 2013. 